\title{
Spin Polarization in Low-Energy Electron Diffraction from Au(110): Theory and Experiment
}

\author{
R. Feder \\ Institut für Festkörperforschung der KFA Jülich, Jülich \\ N. Müller* \\ MPI für Plasmaphysik, EURATOM-Association, Garching \\ D. Wolf* \\ Institut für Kristallographie der Universität München, München
}

Received August 18, 1977

\begin{abstract}
A special LEED system has been constructed, in which the gun is movable in front of the grids and diffracted beams can be extracted through a probe hole in the screen. The polarization is subsequently measured by means of Mott scattering. The $\mathrm{Au}(110)$ surface is of particular interest, because it shows a reversible structure transition, which manifests itself in LEED intensities as a change from a $(1 \times 2)$ pattern at low temperatures to a $(1$ $\times 1)$ pattern at higher temperatures. For the $(1 \times 1)$ structure, spin polarization was measured, with values up to $70 \%$, and calculated for a number of beams as a function of energy and scattering angle. Theoretical and experimental results are in reasonable agreement. In particular, the polarization is found to depend sensitively on geometrical, electronic and vibronic properties of the surface.
\end{abstract}

\section{Introduction}

Within recent years, low-energy electron diffraction (LEED) has become a useful method for determining the structure of clean and adsorbate-covered solid surfaces on the basis of measuring the intensities of diffracted electron beams as functions of energy and orientation of the primary beam [1]. For heavy (large-Z) materials, however, spin-orbit coupling leads to strong spin polarization effects, which could provide additional information: Extrapolating from results on electron-atom scattering [2] such effects were anticipated for solid surfaces [3] and first observed at polycrystalline $\mathrm{W}, \mathrm{Hg}$ and $\mathrm{Au}$ [4]. For diffraction from single crystals, theories were developed $[5,6]$, and sizeable spin polarization effects were measured for $W(001)[7]$, and $\mathrm{Au}(110)$ [8]. Subsequent calculations for W(001) gave encouraging agreement with the experimental data and predicted that spin-polarized low-energy electron diffraction

* The experimental work was sponsored by the Deutsche Forschungsgemeinschaft, Sonderforschungsbereich 128
(SPLEED) could be very sensitive to structural changes $[9,10]$.

The (110) surface of $\mathrm{Au}$ is of particular physical interest because of a structural phase transition, which manisfests itselfs in LEED intensities in a transition from a $(1 \times 2)$ pattern at low temperatures to a $(1 \times 1)$ pattern at higher temperatures $[11,12]$. In the present work, spin polarization measurements from the high temperature phase and theoretical results from an unreconstructed $A u(110)$ surface are reported and compared with each other.

\section{Experimental Set-up}

The experimental set up is schematically shown in Figure 1. The LEED system is a spherical two grid system. The crystal is mounted on a two-axis manipulator. The first axis is in the surface plane of the crystal, i.e. in Figure 1 perpendicular to the plane of 


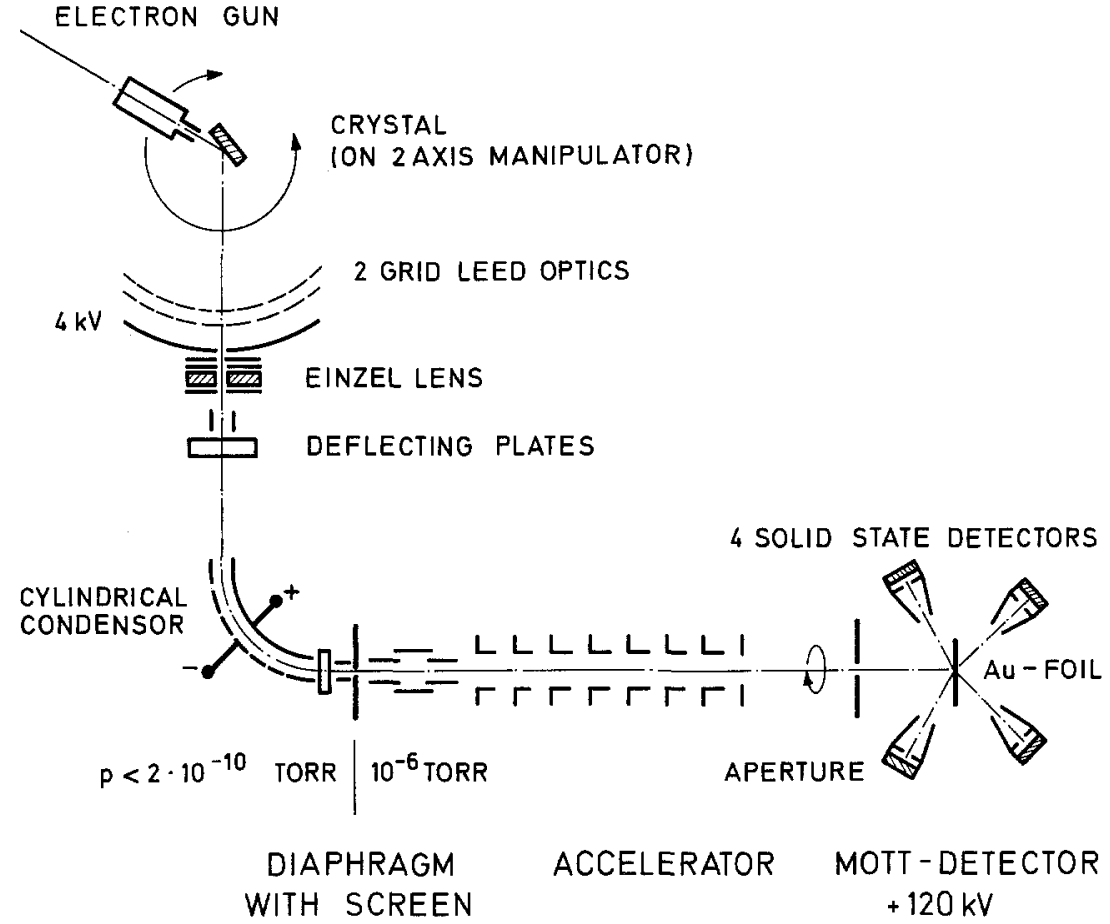

Fig. 1. Experimental set-up for SPLEED drawing, the second one perpendicular to the surface plane. The gun can be rotated in front of the grids about the first axis in an angular range of about $270^{\circ}$. By appropriately choosing the positions of gun and crystal, all beams for which the scattering plane is normal to the first axis can be extracted through a probe hole in the screen. After passing through an imaging and steering system, the beam is deflected by a cylindrical condensor into the polarization detector. The energy discrimination is performed using the grids as a high pass. The cylindrical condensor prevents that gas atoms can get directly from the bad vacuum in the detector to the crystal [13].

The polarization is analysed by means of Mott scattering at a gold foil of $170 \mu \mathrm{g} \mathrm{cm}^{-2}$ in a four counter arrangement. Two counters at a scattering angle of $120^{\circ}$ are sensitive to the spin polarization, the other two at $45^{\circ}$ control apparatus asymmetries.

The measured polarization contains the effect of a background superposition. Denoting by $I_{\uparrow}^{c}\left(I_{\downarrow}^{c}\right)$ the intensity of spin up (spin down) electrons in the coherently diffracted beam and by $I_{\uparrow}^{b}\left(I_{\downarrow}^{b}\right)$ an incoherent background intensity, the observed polarization can be expressed as

$$
P=\left(I_{\uparrow}^{c}-I_{\downarrow}^{c}+I_{\uparrow}^{b}-I_{\downarrow}^{b}\right) /\left(I_{\uparrow}^{c}+I_{\downarrow}^{c}+I_{\uparrow}^{b}+I_{\downarrow}^{b}\right)
$$

If the background is assumed as unpolarized $\left(I_{\uparrow}^{b}=I_{\downarrow}^{b}\right)$, which is not generally true, $P$ is reduced strongly if the background intensity is of the order of magnitude of the coherent intensity. Since thermal diffuse background is large at the high temperatures used in this work, polarization results at diffraction conditions, where the intensity is low, are therefore less reliable. Since the apparatus is not equipped for a proper measurement of beam intensities, we record instead the counting rate in the polarization detector. In doing this, we are aware of two sources of error. Firstly, the probe hole has an acceptance angle of less than $0.5^{\circ}$, whilst, due to surface disorder, the diffracted beams have an angular width up to $3^{\circ}$, which varies with energy and scattering angle (cf. e.g. Ref. 12). Secondly, further electrons are lost on the way to the detector, the number of which depends on the quality of the beam alignment. Bearing these restrictions in mind, we refer, for simplicity, to the counting rates as experimental intensities as opposed to theoretical intensities.

For the case of normal incidence, which is important for determining the real inner potential, the required nonspecular polarization profiles can, in principle, be obtained by orienting the gun normal to the crystal. Since the direction of the detector is, however, fixed with respect to the LEED chamber, this procedure involves, for each energy, a simultaneous angle adjustment of both gun and crystal such that the beam under consideration leaves the crystal in the direction of the detector. The errors, which would result from adjusting these two angles, can be avoided by making use of the reciprocity theorem [14]. The surface nor- 
mal then remains fixed in the direction of the detector, and the gun is aligned in the direction, in which the nonspecular beam would leave the crystal for normal incidence. This gun alignment can be made very precise simply by checking that the normally diffracted beam is centred on the probe hole in the fluorescent screen. For off-normal incidence, the geometrical relation between a diffracted beam and the primary beam is characterized in the present work by the scattering angle $\Theta$ (cf. Fig. 2) rather than the theoretically equivalent polar angle of incidence $\vartheta_{0}$, since $\Theta$ can be measured directly and therefore more accurately.

The surface was prepared as follows. In the first stage, the crystal was bombarded by argon ions $(0.3-3 \mathrm{keV})$ at a variety of angles and annealed at temperatures up to $800^{\circ} \mathrm{C}$. In the second stage, when the contamination was already reduced, neon ions (about $300 \mathrm{eV}$ ) were used at oblique incidence [15]. Subsequent annealing at less than $300^{\circ} \mathrm{C}$ was found to be sufficient to produce a sharp $(1 \times 2)$ LEED pattern.

\section{Theory}

For an unpolarized primary beam, which is normalized to unit intensity, spin polarization and intensity of the elastically diffracted beams have been calculated as functions of energy and orientation of the primary beam by means of a relativistic LEED theory, which has been presented elsewhere $[6,9]$. The surface reciprocal lattice vectors included in the computation were automatically selected such as to ensure convergence. As for the number of monatomic layers, eight were found to suffice. Real and imaginary part of the inner potential were assumed as constants, the former being determined from comparison with experiment, the latter taken as $4 \mathrm{eV}$. For the surface potential barrier, essentially two models were used: firstly, a refracting but non-reflecting barrier, secondly, an exponential-type smooth function. The spacing between the top layer of atoms and the adjacent layer was varied between the bulk value and a contraction of $10 \%$ [16].

The scattering from a single ion-core is described by spin-up and spin-down partial wave shifts (up to $l$ $=7$ ), which were obtained from a muffintin bandstructure potential [17]. Since comparison is intended with experimental data obtained at high temperature $(710<T<750 \mathrm{~K})$ the incorporation of thermal effects in the calculation requires particular attention. Thermal lattice expansion has been neglected, since its effect on polarization results was found to be negligible compared to thermal lattice vibrations. These have been incorporated - as has so far been customary in LEED [1] - by averaging the atomic scattering amplitudes over a Debye spectrum, which amounts to replacing the actual real phase shifts by effective
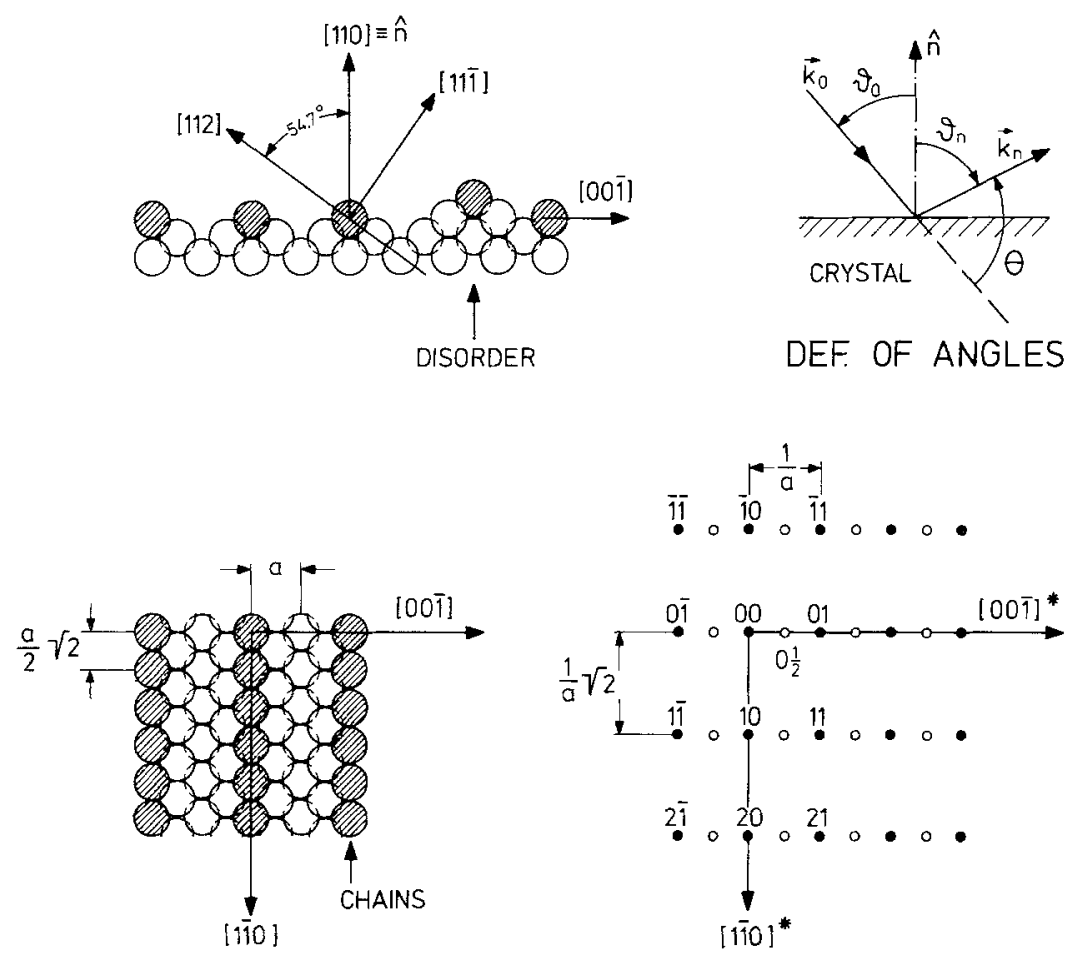

SURFACE LATTICE
Fig. 2. Crystal surface geometry of $A u(110)$, nomenclature in the reciprocal lattice and definition of angles in the diffraction process. The surface is shown with the $(1 \times 2)$ superstructure. Heating the crystal will bring disorder and decay to the hatched chains. The half order points in the reciprocal lattice will then disappear 


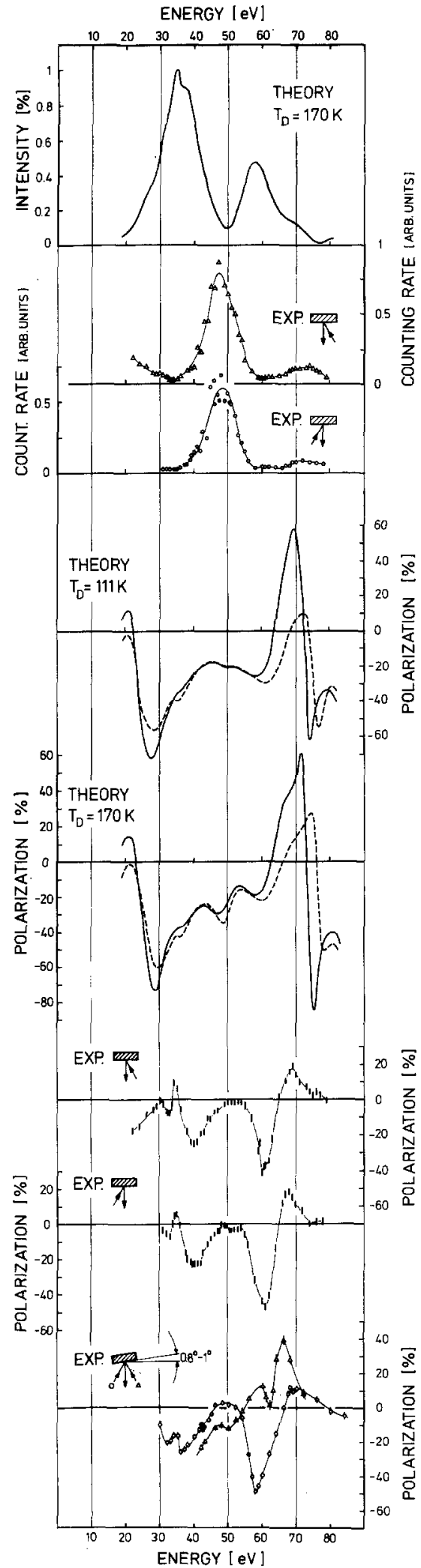

Fig. 3. 10 beam: intensity, counting rate and polarization for normal incidence as functions of energy ( $-0 \%$ contraction, ----$5 \%$ contraction); theoretical curves for crystal temperature $T$ $=723 \mathrm{~K}$, experimental curves for $T=710-750 \mathrm{~K}$. The error bars give the statistical errors. Additional experimental uncertainties are: energy $\pm 1 \mathrm{eV}$, polarization zero $\pm 0.5 \%$, polarization calibration $\pm 5 \%$ of given values complex phase shifts. Correlations between the atomic vibrations are neglected. For the Debye temperature, which enters at this stage, the sophistication of using different values for different monatomic layers seems not warranted at present. Instead, we use for all layers the extreme values of the bulk $\left(T_{D}=170 \mathrm{~K}\right)$ $[18]$ and the surface Debye temperature $\left(T_{D}=111 \mathrm{~K}\right)$ [19]. The crystal temperature was taken as $723 \mathrm{~K}$.

\section{Results and Discussion}

In view of determining the real inner potential to be used in the calculations, it is appropriate to first investigate the case of normal incidence (of the primary beam), in which the inner potential $V_{0}$ is essentially the shift between experimental intensity or polarization versus energy profiles and their theoretical counterparts calculated for $V_{0}=0$. Concentrating on non-specular beams adds the advantage of less sensitivity to variations in the surface barrier model.

For the beam indexing (nomenclature) used in the present work we refer to the schematic diffraction pattern in Figure 2. Spin polarization and intensity versus energy results for normal incidence are shown in Figure 3 for the 10-beam and in Figure 4 for the 11beam. Comparing experiment and theory-shown with an inner potential shift of $14 \mathrm{eV}$-for the polarization of the 10-beam, there are substantial discrepancies in the curve shapes and absolute values, but the positions in energy of the extrema (positive and negative peaks) match within a few $\mathrm{eV}$. The intensity profiles would fit reasonably well if one assumed an inner potential of $4 \mathrm{eV}$. Such a low value seems, however, physically unreasonable, apart from being inconsistent with the above $14 \mathrm{eV}$. We have, at the moment, no proper explanation for this inconsistency, but surmise that structural properties of the real surface, which are not included in the theoretical model, are responsible. As a particular interesting result we found that the polarization profiles, both experimental and theoretical, are extremely sensitive to very small variations (of the order of $1^{\circ}$ ) in the angle of incidence. For the experiment, this implies that the beam adjustment must be extremely accurate. Special checks should therefore be made using the two-fold symmetry of the surface. In our method of measuring nonspecular beams for normal incidence via reciprocity (cf. Section 2), this is achieved by interchanging the two symmetrically equivalent (oblique) directions of incidence. If there is no magnetic field and if the primary beam impinges - for each energy - on the same surface region in both cases, equivalence of the two measured profiles is a necessary and sufficient condition. But even if these two 
sources of error cannot be excluded entirely, as is the case in our experiment, it still guarantees reliability of the data.

For the 11-beam-Figure 4-the experimental polarization profile is found to agree well with profiles calculated for a Debye temperature $T_{D}=170 \mathrm{~K}$ provided that one applies an inner potential shift $V_{0}$ of $14 \mathrm{eV}$ near $50 \mathrm{eV}$ and an increasingly smaller one at higher energies. Such an energy dependence of $V_{0}$ is in accordance with other LEED results [20]. As for a possible contraction of the top layer spacing, Figure 4 suggests that it is closer to 0 than to $5 \%$. The results obtained for $T_{D}=111 \mathrm{~K}$ show less structure and agree less well with experiment. This appears plausible on the grounds that, at normal incidence, bulk layers, for which $T_{D}=170 \mathrm{~K}$ is more appropriate, contribute strongly to the scattering amplitudes. Calculations for different surface barriers are found to be practically identical. The excellent agreement between the two measured polarization profiles is evidence of the reliability of the experiment.

The inner potential value of $14 \mathrm{eV}$ found in Figure 4 was used in further calculations at constant energy $E$ $=50 \mathrm{eV}$ for incidence in an azimuth normal to the "chains" of the surface net, varying the scattering angle $\Theta$ (for definition of angles see Figure 2).

In Figure 5 we present polarization and intensity versus $\Theta$ profiles for the 01-beam. For scattering angles $\Theta$ larger than approximately $115^{\circ}$, i.e. in a range, in which the directions of the primary and the diffracted beam are close to the surface normal, the experimental polarization profile is in excellent agreement with theoretical profiles for $T_{D}=170 \mathrm{~K}$ and a slight contraction of the top spacing. The values of these parameters are consistent with the results from Figure 4. The intensities in this angular range are in good agreement. For $\Theta$ smaller than $115^{\circ}$, we find still excellent polarization agreement near $100^{\circ}$, but two significant discrepancies near $110^{\circ}$ andbelow $90^{\circ}$. It is interesting to note that the experimental $110^{\circ}$ polarization feature is correlated with a drastic drop in the experimental intensity, and that the latter remains very low in the entire region. As a possible reason for the discrepancies we suggest the fact that the real surface exhibits strong disorder and possibly remnants of a superstructure, whilst the calculations apply to an ideal perfect surface. We further note that for the $110^{\circ}$ feature the direction $\mathbf{k}_{n}$ of the diffracted beam nearly coincides with the inclination of the (111) facets.

Polarization and intensity versus $\Theta$ profiles for the 00-beam are shown in Figure 6 . In contrast to the nonspecular beams, the calculated results for the specular beam are found to be very sensitive to the surface barrier model, in addition to the Debye tem-

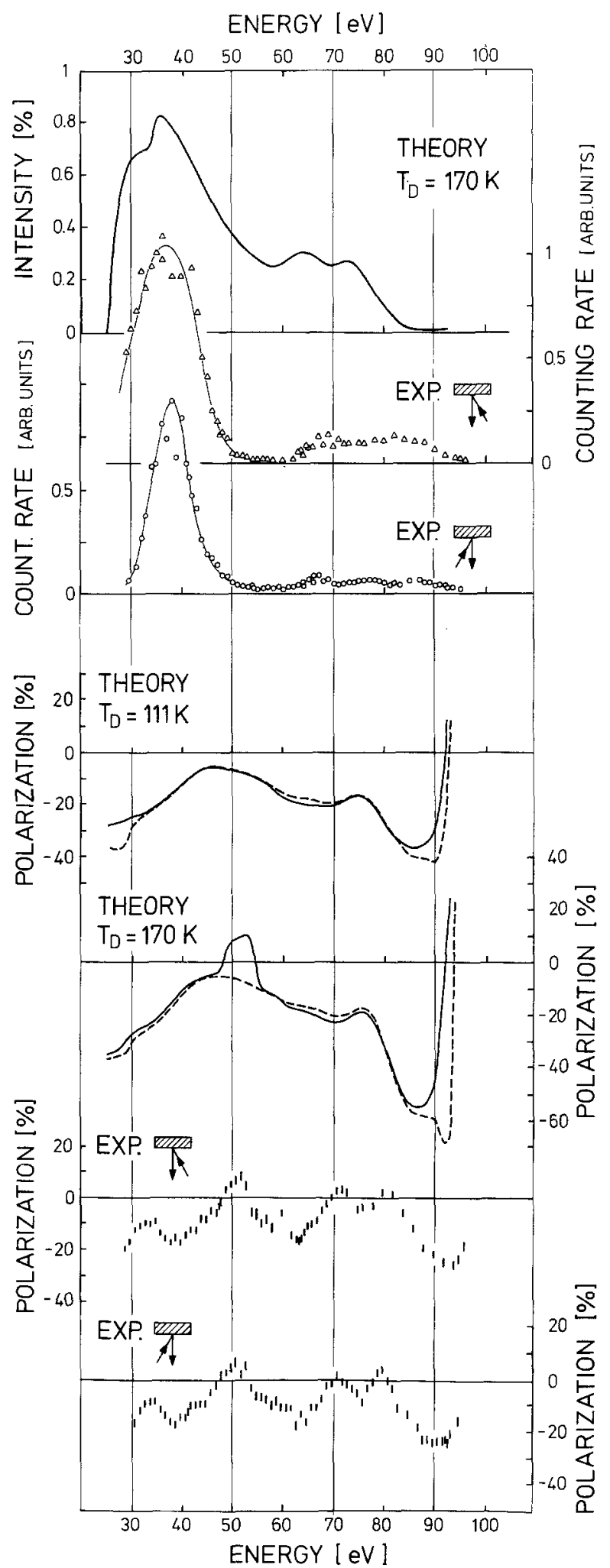

Fig. 4. 11 beam: intensity, counting rate and polarization for normal incidence as functions of energy (- $-0 \%$ contraction, -... $5 \%$ contraction); theoretical curves for crystal temperature $T$ $=723 \mathrm{~K}$, experimental curves for $T=710-750 \mathrm{~K}$. For experimental errors see Figure 3. Since the scattering plane is not a mirror plane, the length of the polarization vector differs from its normal component. 'Polarization' refers to the latter 
SCATTERING ANGLE $\theta$

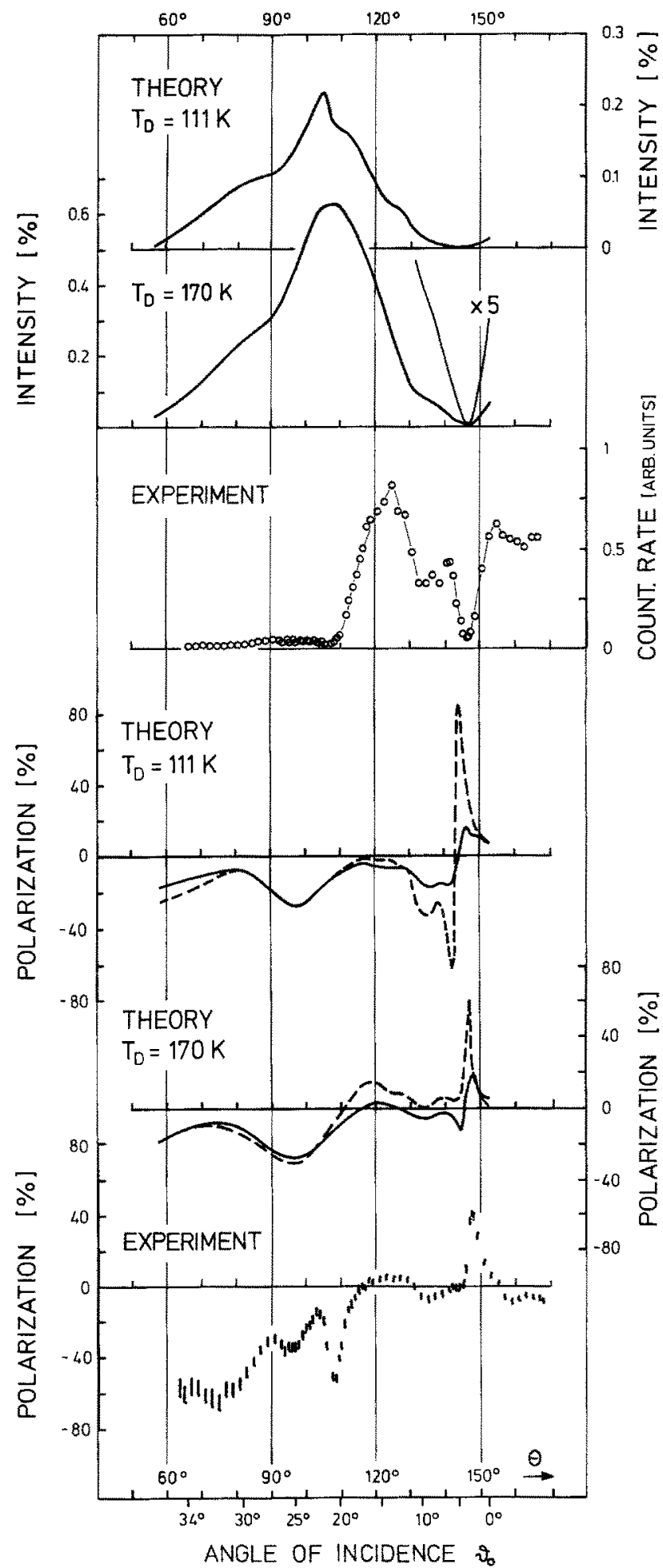

Fig. 5. 01 beam: intensity, counting rate and polarization for $E$ $=50 \mathrm{eV}$ as functions of the scattering angle $\Theta(-0 \%$ contraction, -.-- $10 \%$ contraction); theoretical curves for crystal temperature $T=723 \mathrm{~K}$, experimental curves for $T=710-750 \mathrm{~K}$, primary beam normal to $1 \overline{1} 0$ direction. The error bars give the statistical errors. Additional experimental uncertainties are scattering angle $\pm 2^{\circ}$, polarization zero $\pm 0.5 \%$, polarization calibration $\pm 5 \%$ of given values, energy $\pm 1 \mathrm{eV}$

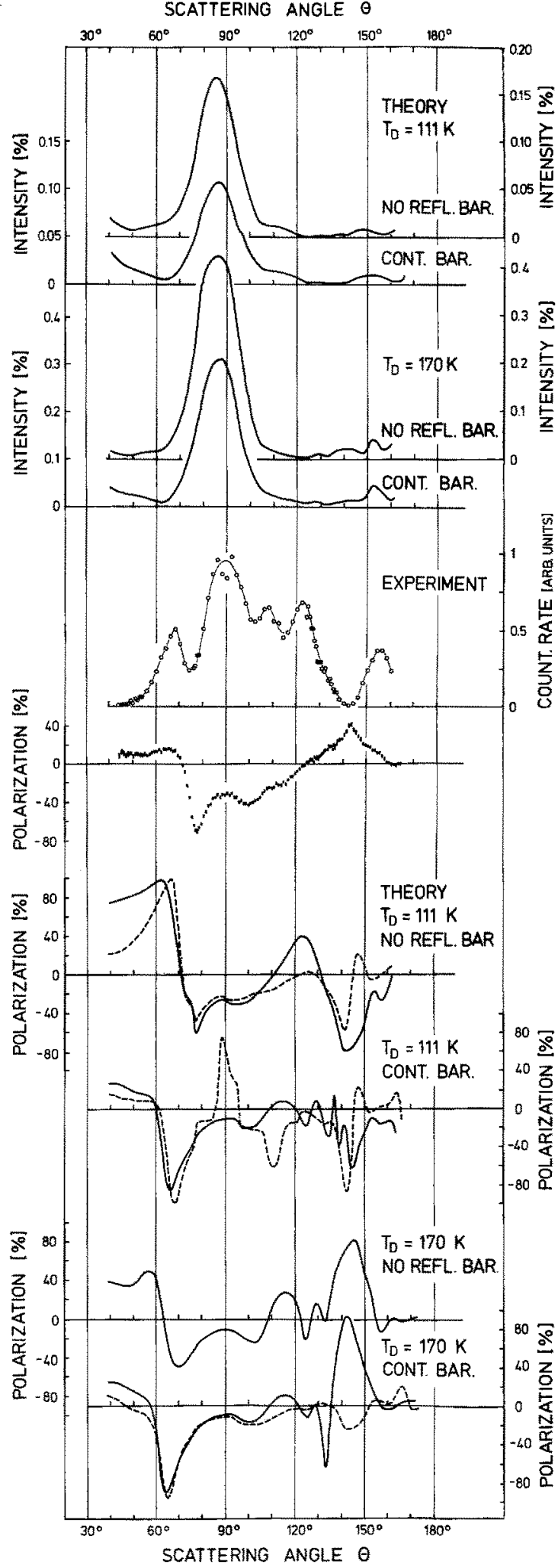

Fig. 6. 00 beam: intensity, counting rate and polarization for $E$ $=50 \mathrm{eV}$ as functions of the scattering angle $\Theta(-0 \%$ con traction, - - $10 \%$ contraction); theoretical curves for crystal temperature $T=723 \mathrm{~K}$, experimental curves for $T=710-750 \mathrm{~K}$. The primary beam is normal to 110 . For experimental errors see Figure 5 
perature and the contraction of the top layer spacing. Comparison between $0 \%$ and $10 \%$ contraction results reveals significant differences. For the $T_{D}=111$ and $170 \mathrm{~K}$ comparison, particular attention is drawn to the regime of $\Theta$ near $140^{\circ}$. For $\Theta<80^{\circ}$, i.e. for very oblique incidence, the barrier outweighs the influence of a contraction. Comparing theory and experiment, we mostly find very good agreement for $T_{D}=111 \mathrm{~K}$. The somewhat surprising preference of the no-reflection barrier for $\Theta>70^{\circ}$ over a continuous one might be ascribed to the disorder of the surface. Below $\Theta=70^{\circ}$, the smooth barrier apparenly yields a better fit. This should, however, be seen in conjunction with the substantial increase in experimental intensity near $\Theta=70^{\circ}\left(\vartheta_{0}=\vartheta_{n}=55^{\circ}\right)$, and interpreted similarly as the $\Theta<110^{\circ}$ regime of Figure 5 .

\section{Conclusion}

Theoretical polarization results have been found to be sensitive to contraction of the top layer spacing, to the surface barrier and to thermal lattice vibrations. Large spin polarization effects have been measured, and the agreement between theory and experiment is generally good, so that comparison can provide information about the above properties as well as clues to deviations of the real surface structure from the theoretical model. Since the $A u(110)$ surface-at the temperature required for a $(1 \times 1)$ LEED pattern exhibits considerable disorder as well as remnants of a superstructure, and since the diffuse background intensity can be very strong, more detailed calculations are, however, not warranted at the present stage [21]. Bearing this in mind, we feel that our results are most encouraging in view of harnessing SPLEED as a sensitive tool for surface structure analysis.

We are very grateful to $H$. Plöckl and G. Nagleder for valuable technical help, to Dipl. Phys. K. Ertl, Dipl. Ing. P. Bauer, and Ing. grad. U. Dinklage for assisting with the experiment.

Particular thanks are due to Prof. Dr. G. Eilenberger for greatly encouraging collaboration between theory and experiment. We are also grateful to Dr. W. Eckstein, Dr. H. Vernickel, and Prof. Dr. H. Jagodzinski for promoting the cooperation of different institutes.

\section{References}

1. For a recent review and further references see e.g. Jona, F. and Marcus, P.M.: Comments Sol. State Phys. 8, 1 (1977)

2. Kessler, J.: Polarized Electrons. Berlin-Heidelberg-New York: Springer 1976

3. Maison, D.: Phys. Letters 19, 654 (1966)

4. Eckstein, W.: Z. Physik 203, 59 (1967); Loth, R.: Z. Physik 203, $66(1967)$
5. Jennings, P.J.: Surface Sci. 20, 18 (1970)

6. Feder, R.: phys. stat. sol. (b) 46, K 31 (1971) and 49, 699 (1972)

7. O'Neill, M.R., Kalisvaart, M., Dunning, F.B., Walters, G.K.: Phys. Rev. Letters 34, 1167 (1975)

Kalisvaart, M., Riddle, T.W., Dunning, F.B., Walters, G.K.: Conf. Physical Electronics, June 1977

8. Müller, N. and Wolf, D.: Bull. Am. Phys. Soc. II 21, 944 (1976)

9. Feder, R.: Phys. Rev. Letters 36, 598 (1976)

10. Feder, R.; Surface Sci. 63, 283 (1977) and 'to be published'

11. Fedak, D.G., Gjostein, N.A.: Acta Met. 15, 827 (1967), and Surface Sci. 8, 77 (1967)

12. Wolf, D.: Dissertation (München 1972), and Wolf, D., Jagodzinski, H., Moritz, W.: to be published in Surface Sci. (part III and IV of a series of papers)

13. The cylindrical condensor was essential in an earlier design used for field emission (Müller, N., Eckstein, W., Heiland, W. Zinn, W., Phys. Rev. Letters 29, 1651 (1972). It should be noted that it does not affect a spin polarization vector normal to the scattering plane.

14. Woodruff, D.P., Holland, B.W.: Physics Letters 31 A, 207 (1970);

Lagally, M.G., Ngoc, T.C., Webb, M.B.: Surface Sci. 25, 444 (1971)

15. Heiland, W., Taglauer, E.: private communication; Taglauer, E., Beitat, U., Marin, G., Heiland, W.: J. Nucl. Mat. 63, 193 (1976)

16. Although for a $10 \%$ contraction the nearest-neighbour distance is less than allowed from the point of view of a bulk ionic radius, this case cannot be ruled out a priori. For the diatomic molecule $\mathrm{Au}_{2}$, for example, the internuclear distance was found to be significantly less than twice the ionic radius (Huber, K.P.: American Institute of Physics Handbook, Section 7g. New York: McGraw Hill 1972)

17. Andersen, O.K.: private communication

18. International tables for X-ray crystallography, vol. III

19. Jackson, D.P.: Surface Sci. 43, 431 (1974)

20. cf. e.g. Jepsen, D.W., Marcus, P.M., Jona F.: Phys. Rev. B5, $3933(1972)$

21. It seems pertinent to note that for other fcc(110) surfaces, such as $\mathrm{Al}$ and $\mathrm{Ag}$, attempts to determine the surface structure via LEED intensities, have not been entirely satisfactory either (cf. Zanazzi, E., Jona, F., Jepsen, D.W., Marcus, P.M.: J. Phys. C10, 375 (1977))

\section{R. Feder}

Institut für Festkörperforschung

Kernforschungsanlage Jülich $\mathrm{GmbH}$

Postfach 1913

D-5170 Jülich 1

Federal Republic of Germany

N. Müller

Max-Planck-Institut

für Plasmaphysik

EURATOM-Association

D-8046 Garching bei München

Federal Republic of Germany

D. Wolf

Institut für Kristallographie

der Universität München

Theresienstraße 41

D-8000 München 2

Federal Republic of Germany 